\title{
Characterisation of follicular dendritic cells in labial salivary glands of patients with primary Sjogren syndrome: comparison with tonsillar lymphoid follicles
}

Karim Elias Aziz, Peter J McCluskey, Denis Wakefield

\begin{abstract}
Objective-To localise and characterise follicular dendritic cells (FDC) present in autoimmune lesions of primary Sjogren syndrome.

Methods-Cryostat sections of labial salivary glands from 15 patients with primary Sjogren syndrome were examined by an indirect immunoperoxidase technique and monoclonal antibodies to a panel of dendritic cell markers. Tonsils from two controls were also examined for the same markers.

Results-FDC were localised in the centre of $75 \%$ of lymphoid focal structures in labial salivary gland biopsies. FDC in labial salivary glands of patients with primary Sjogren syndrome expressed CD35, CD11c, and CD106 (VCAM-1) in a pattern similar to FDC in tonsils, but they did not express either CD14 or CD11b. This indicates that they may not be of myeloid origin, while FDC in tonsillar lymphoid follicles strongly expressed both CD14 and CD11b. FDC in labial salivary glands of patients also lacked VLA-2 $\alpha$ and VLA-3 $\alpha$, which were expressed by FDC in tonsils. Conclusions-The characteristic phenotype and origin of these cells may be of importance in the immune responses involved in Sjogren syndrome and the retention of infiltrating lymphocytes in the glands.
\end{abstract}

(Ann Rheum Dis 1997;56:140-143)

Primary Sjogren syndrome is a chronic inflammatory disorder associated with lymphocytic infiltration of exocrine glands. ${ }^{1-3}$ The infiltrate is composed mainly of $\mathrm{T}$ lymphocytes, ${ }^{23}$ though in some labial salivary biopsies non-T/ non-B lymphocytes predominate. ${ }^{4}$ However, CD16 natural killer (NK) lymphocytes are rarely seen. ${ }^{5}$ The lymphocytic infiltration is progressive $^{6}$ and the infiltrating lymphocytes are retained and persist in the exocrine glands. ${ }^{57}$ Several autoantibodies have been shown to be associated with Sjogren syndrome (SS) (including rheumatoid factor, anti-SS-A, and anti-SS-B). ${ }^{2}$ The disease is not associated with any cellular proliferative responses to self antigens. ${ }^{8}$ Hence there is no explanation as to what keeps the infiltrating lymphocytes in the glands. Several viruses have been proposed as triggers of the autoimmune response. ${ }^{7} 9$ The mechanisms involved in the retention of infiltrating lymphocytes in the glands may involve changes in the pattern or density of adhesion molecule expression. ${ }^{5}$ Another possibility is the presence of follicular dendritic cells (FDC) which may functionally maintain the lymphocytes in lymphoid follicles, as seen in the salivary or lacrimal glands of these patients. The aim of this study was to compare the phenotypic characteristics of FDC in labial salivary glands of patients with primary Sjogren syndrome and those seen in tonsillar lymphoid follicles, as an example of organised peripheral lymphoid tissue. This may increase our understanding of Sjogren syndrome.

The origins of dendritic cells are not completely clear. ${ }^{10}{ }^{11}$ Dendritic cells can form clusters with T or B lymphocytes. ${ }^{1011}$ They may stimulate autologous and allogeneic mixed leucocyte reactions. ${ }^{1011}$ They are powerful accessory cells and can induce the expression of activation antigens by lymphocytes. ${ }^{10}{ }^{11}$ Dendritic cells are situated in close proximity to lymphocytes in lymphoid organs. This rendered the determination of their antigenic phenotype difficult. ${ }^{111}$ There is no accepted classification of dendritic cells, and most classifications describe a phenotypic and functional overlap between different types of dendritic cell. ${ }^{10-13}$ CD35 (DRC-1) may be expressed on all dendritic cells in lymphoid tissues. ${ }^{12}$ Schriever et al isolated unclustered FDC and phenotypically characterised these cells. ${ }^{10}$ They reported that FDC express several complement receptors (CR), namely CR1 (CD35), CR2 (CD21), CR3 (CD11b), and the myeloid/monocytic cell marker CD14. Another group showed that isolated FDC lack the expression of CD35. ${ }^{11}$ This may be due to the isolation of a different subpopulation of dendritic cells from the same lymphoid organ resulting from variable origins of the dendritic cells, ${ }^{14}$ variable stages of maturation, or functional alteration of these cells in different physiological or pathological situations. ${ }^{15}$

VLA- $2 \alpha$ and VLA- $3 \alpha$ are integrin molecules that may be expressed by FDC ${ }^{10116}$ and they have a common $\beta$ chain CD29. ${ }^{5}$ The ligands for VLA- $2 \alpha$ and VLA- $3 \alpha$ are laminin, collagen, and fibronectin. 


\section{Methods}

SUBJECTS

Fifteen patients with primary Sjogren syndrome fulfilling at least five (a minimum of four is required) of the European Community criteria ${ }^{1}$ were the subjects of this study. Patients ranged in age from 24 to 87 years, with a mean of 50.4 years. There were 14 females and one male. A positive Schirmer test was demonstrated in 11 patients $(73 \%)$ and a positive Rose Bengal test in 14 (93\%). Abnormal unstimulated salivary flow rates $(<0.1 \mathrm{ml}$ $\mathrm{min}^{-1}$ ) were found in 12 patients $(80 \%)$. Rheumatoid factor was detected in 12 patients $(80 \%)$, anti-nuclear antibodies (ANA) in 11 $(73 \%)$, and anti-SS-A and/or anti-SS-B in nine $(60 \%)$. Serum anti-ds-DNA was not detected in any of the patients. All labial salivary gland biopsies of patients with primary Sjogren syndrome included in this study had two or more lymphoid focal structures per $4 \mathrm{~mm}^{2}$ (grade IV). ${ }^{17}$

Ethics committee approval was obtained. All subjects gave informed consent to their participation in the study. Two tonsils were obtained from two female patients undergoing routine tonsillectomy at Prince of Wales Hospital, Sydney.

LABIAL SALIVARY GLAND BIOPSY

The technique used for obtaining labial salivary glands from patients was based on previously described methods. ${ }^{9}$ Labial salivary gland and tonsil specimens were transferred in normal saline $(0.9 \% \mathrm{NaCl})$ to be used for immunohistology. The biopsies were embedded in OCT compound (Bayer, Miles Laboratories), snap frozen, and cryostat sections 4 microns thick were cut. The sections were fixed in cold $\left(2-4^{\circ} \mathrm{C}\right)$ acetone for five minutes followed by phosphate buffered saline (PBS) for five minutes (repeated twice in PBS). Sections were stored at $-70^{\circ} \mathrm{C}$.

IMMUNOHISTOCHEMISTRY

An indirect immunoperoxidase technique was used as described before. ${ }^{5}$ The specificities and dilutions of the monoclonal antibodies which were used as primary antibodies are listed in table 1 .
MICROSCOPIC EXAMINATION OF SECTIONS Sections were examined with light microscopy using a Microphot FX microscope (Nikon, Japan). Labial salivary gland sections were examined for positive staining as indicated by the brown colour developed by the specific immunohistochemical reaction. Follicular dendritic cells were localised as shown by positive staining with CD35 (DRC-1), CD106 (VCAM-1), and their characteristic dendritic morphology. Other cellular elements staining with each antibody were recorded.

\section{Results}

EXPRESSION OF DENDRITIC CELL MARKERS BY FDC IN LABIAL SALIVARY GLANDS OF PATIENTS WITH PRIMARY SJOGREN SYNDROME

FDC in labial salivary glands of patients with primary Sjogren syndrome expressed CD35 (DRC 1) to the same extent as in tonsillar sections (figure, A, B, table 2). VLA-2 $\alpha$ (CD49b) and VLA-3 $\alpha$ (CD49c) were not expressed by follicular dendritic cells in labial salivary glands of patients with Sjogren syndrome (table 2), while FDC (in situ) in lymphoid follicles of tonsils expressed both VLA-2 $\alpha$ and VLA- $3 \alpha$ strongly, although at a lesser intensity than CD35 or CD11c (figure, C, D, table 2). FDC in labial salivary glands and tonsils expressed CD29 ( $\beta-1$ integrin), the $\beta$ chain for VLA-1, $-2,-3,-4,-5$, and -6 molecules. Acinar and ductal epithelial cells in labial salivary glands of patients with primary Sjogren syndrome expressed both VLA-2 $\alpha$ and VLA-3 $\alpha$ (figure, D). Similarly, stratified squamous epithelial cells in tonsils expressed both antigens. VCAM-1 was detectable at a variable intensity (from one follicle to another) on FDC in labial salivary glands of patients with primary Sjogren syndrome (figure, $\mathrm{H}$ ) and in tonsils (table 2). CD11c was strongly expressed by FDC both from labial salivary glands of patients and from tonsils (figure, F, G). Neither CD11b nor CD14 was identifiable on dendritic cells in labial salivary glands of patients with primary Sjogren syndrome. FDC in tonsillar lymphoid follicles expressed CD11b and CD14 (figure, E). Although this study shows the expression of CD35, CD11b, and CD14 on FDC in tonsils, it cannot

Table 1 Mouse monoclonal antibodies used in this study

\begin{tabular}{|c|c|c|c|c|}
\hline Antigen/specificity & Clone & Ig chain & Supplier & Concentration \\
\hline \multicolumn{5}{|l|}{ Dendritic cell markers: } \\
\hline CD35 (CR1, DRC-1) & Ber-MAC-DRC & IgG & Dako & $1 / 50$ \\
\hline CD29 ( $\beta 1)$ & 4B4LDC9LDH8 & IgG1 & Coulter & $1 / 50$ \\
\hline CD $49 \mathrm{~b}(\alpha 2$, VLA-2 $\alpha)$ & P1E6 & IgG1 & Dako & $1 / 50$ \\
\hline CD $49 \mathrm{c}(\alpha 3$, VLA $-3 \alpha)$ & P1B5 & IgG1 & Dako & $1 / 50$ \\
\hline CD18 ( $\beta 2$ integrin) & MHM23 & IgG1 & Dako & $1 / 50$ \\
\hline CD11a $(\alpha \mathrm{L}, \mathrm{LFA}-1 \alpha)$ & MHM24 & IgG1 & Dako & $1 / 50$ \\
\hline $\operatorname{CD} 11 \mathrm{~b}(\alpha \mathrm{M}, \mathrm{C} 3 \mathrm{biR} \alpha)$ & 2LPM19c & IgG1 & Dako & $1 / 50$ \\
\hline CD11c $(\alpha \mathrm{X}, \mathrm{p} 150.95)$ & SHCL3 & IgG2b & Dako & $1 / 50$ \\
\hline VCAM-1 (CD106) & $1 \mathrm{G} 11$ & IgG1 & Immunotech & $1 / 50$ \\
\hline VCAM-1 (SD106) & 2G7 & IgG1 & Otsuka Pharmaceutical & $1 / 100$ \\
\hline CD14 & 116 & $\operatorname{IgM}$ & Coulter & $1 / 50$ \\
\hline Class II-MHC(HLA-DR) & L243 & IgG2a & Becton Dickinson & $1 / 20$ \\
\hline Class I-MHC & W6/32 & IgG2 & Dako & $1 / 50$ \\
\hline ICAM-1 (CD54) & LB-2 & $\operatorname{IgG} 2 b$ & Becton Dickinson & $1 / 20$ \\
\hline
\end{tabular}
receptor-3; $\alpha 2, \alpha 3$ : integrins of $\beta 1$ integrin family; $\alpha \mathrm{X}, \alpha \mathrm{M}$ : integrins of $\beta 2$ family; VCAM-1, vascular cell adhesion molecule-1 (CD106). 
confirm that all dendritic cells in tonsillar lymphoid follicles expressed these markers. Class I and class II major histocompatibility complex (MHC) expression was demonstrated on FDC of patients with primary Sjogren syndrome and on tonsils (table 2). CD11a ( $\alpha \mathrm{L}$ integrin), CD18 ( $\beta-2$ integrin), and their ligand ICAM-1 were also strongly expressed by FDC in labial salivary glands and tonsils (table 2).

Follicular dendritic cells were identified in a considerable proportion of lymphoid follicles present in labial salivary glands of patients with primary Sjogren syndrome. The percentage of lymphoid follicles that have detectable follicular dendritic cells ranged from $29 \%$ to $100 \%$ of follicles in labial salivary glands. The majority of labial salivary glands contained FDC in more than 50\% [mean (SEM) 74.7 (5.9) \%] of the lymphoid follicles present. Tonsil sections showed FDC in $99 \%$ of lymphoid follicles. FDC were sometimes seen in small lymphoid aggregates not forming follicles. FDC were not seen adjacent to glandular epithelium or epimyoepithelial islands.
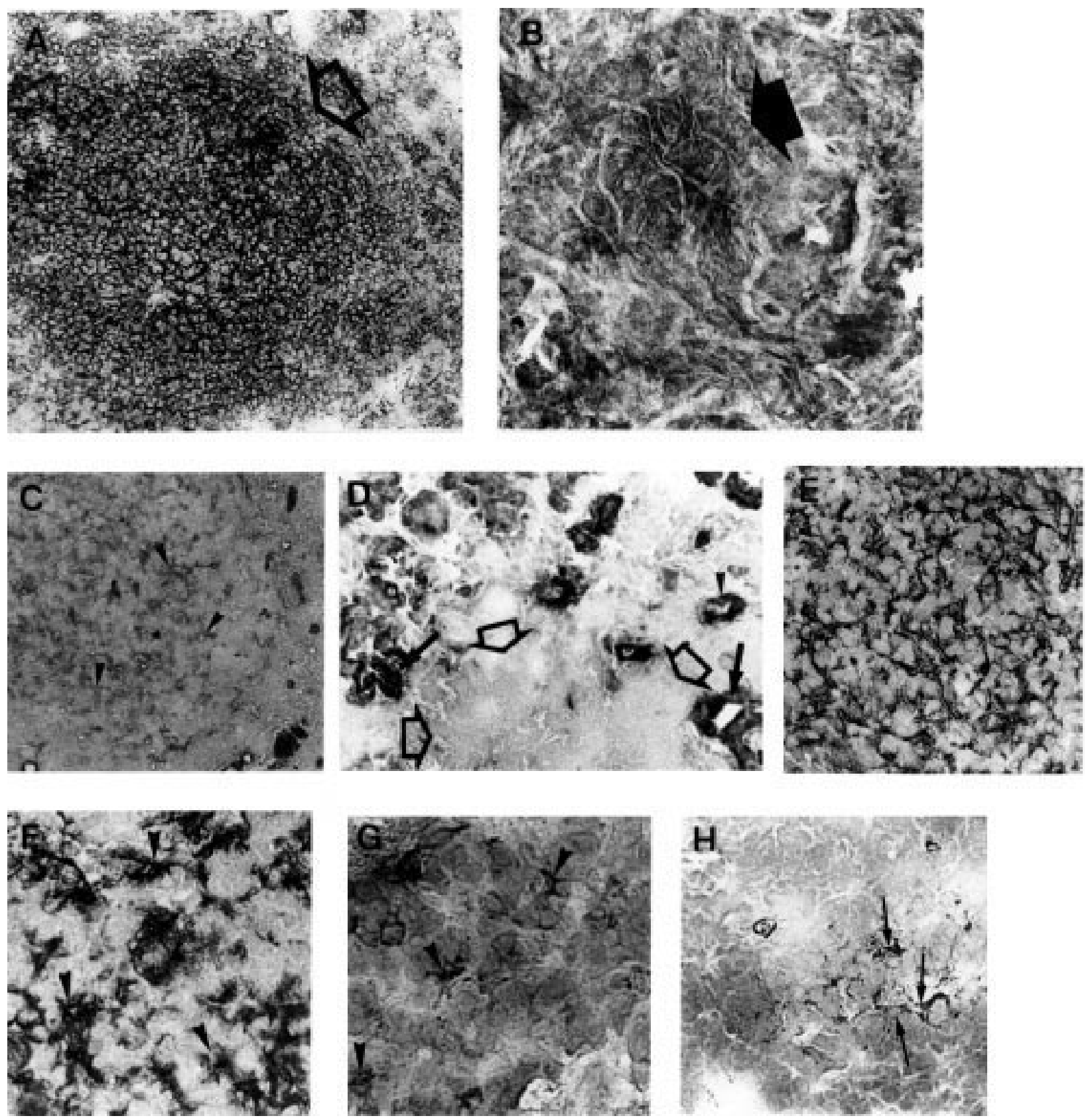

Frozen sections of labial salivary glands of patients with primary Sjogren syndrome and tonsils examined by an indirect immunoperoxidase technique yielding a brown colour which appears as dark shadows in the photomicrographs. (A) Follicular dendritic cells and lymphocytes in the germinal centre (large arrow) of a tonsillar lymphoid follicle intensely express CD35 (×225). (B) Several dendritic cells in that lymphoid follicle (large arrow) in a labial salivary gland express CD35 (×225). (C) Tonsillar dendritic cells (arrow heads) express VLA-2a in the germinal centre ( $\times 113)$. (D) A lymphoid focus (large arrows) shows the absence of VLA-2a while ductal (arrows), acinar epithelial cells, and endothelial cells (arrow head) intensely expressed that molecule in labial salivary glands of a patient with primary Sjogren syndrome $(\times 225)$. That lymphoid focus contained dendritic cells that expressed CD35 and VCAM-1. (E) Dendritic cells express CD11b in tonsils ( $\times 225)$. (F) Dendritic cells (arrow heads) in tonsillar lymphoid follicles intensely express CD11c in that germinal centre $(\times 225)$. (G) Several dendritic cells (arrow heads) in a lymphoid focus in labial salivary glands of a patient with primary Sjogren syndrome express distinctly CD11c (×225). (H) A lymphoid follicle in labial salivary gland shows VCAM-1 expression by dendritic cells (arrows) ( $\times 225)$. 
Table 2 Expression of different dentritic cell markers by follicular dendritic cells (FDC) in labial salivary glands (LSG) of patients with Sjogren syndrome and tonsillar lymphoid follicles

\begin{tabular}{lll}
\hline Marker & FDC, LSG, Sjogren syndrome & FDC, tonsils \\
\hline CD35 (DRC1) & +++ & +++ \\
CD29 (VLA- $\beta$ ) & +++ & +++ \\
CD49b (VLA-2 $\alpha$ ) & Negative & + \\
CD49c (VLA-3 $\alpha)$ & Negative & + \\
CD18 & +++ & +++ \\
CD11a & ++ & ++ \\
CD11c (p150.95) & ++ & ++ \\
CD11b (C3biR) & $(-+)$ & ++++ \\
VCAM-1 (CD106) & $+\rightarrow++$ & + \\
CD14 & Negative & + \\
Class I MHC & + & ++ \\
Class II MHC (DR) & ++ & +++ \\
CD54 (ICAM-1) & +++ & ++ \\
\hline
\end{tabular}

Grades: Negative, not detectable; +/-, detectable on few cells with dendritic cell morphology; +, detectable on a proportion of dendritic cells; ++ , detectable on the majority $(\geq 50 \%)$ of follicular dendritic cells; +++, detectable on almost all follicular dendritic cells.

\section{Discussion}

In this study we showed that FDC were present in lymphoid follicles in labial salivary glands from patients with primary Sjogren syndrome. The expression of CD11c by these FDC may be important. Blocking CD11c has been shown to decrease the stimulatory effect of dendritic cells on $\mathrm{T}$ cell proliferation. ${ }^{1819}$ CD $11 \mathrm{c}$ is expressed by blood dendritic cells ${ }^{18} 19$ and tonsillar FDC. The lack of expression of the myeloid markers CD14 and CD11b by FDC in labial salivary glands of patients with Sjogren syndrome indicates that these cells are more mature and may have more efficient accessory functions, as shown by blood dendritic cells that do not express these markers. ${ }^{15}$ This may also indicate that the dendritic cells in labial salivary glands of patients with Sjogren syndrome may not be of myeloid origin. Different origins for FDC have been proposed, including myeloid or lymphoid progenitors. ${ }^{101415}$ The expression of lymphoid markers, such as CD3, or in some foci CD19, by lymphocytes ${ }^{5}$ in the close proximity of dendritic cells makes definite determination of the expression of these markers by the dendritic cells themselves difficult. Separation of the dendritic cells may allow more satisfactory examination of their phenotypic and functional characteristics, though the small size of the biopsies and small number of dendritic cells present will limit the feasibility of such an in vitro approach. Another interesting finding is the lack of expression of VLA- $2 \alpha$ and VLA-3 $\alpha$ by FDC in labial salivary glands of patients. ${ }^{10} 16$ Although FDC in labial salivary glands and tonsils express VCAM-1, blood dendritic cells do not express this molecule. ${ }^{18}$ If these dendritic cells originated from the blood, they may have acquired VCAM-1 and increased CD11c expression in the local autoimmune lesions in labial salivary glands of patients with Sjogren syndrome.
The results of this study show that dendritic cells are present in labial salivary glands of patients with Sjogren syndrome. They may play an important role in regulating immune responses and the retention of infiltrating lymphocytes in the glands. Although the origin of these dendritic cells is not clear, their phenotypic characteristics suggest that they may have originated from non-myeloid blood dendritic cells. Blood dendritic cells can bind to a number of adhesion molecules ${ }^{18} 19$ that are expressed on endothelial cells in labial salivary glands of patients with Sjogren syndrome ${ }^{5}$ and this may provide a portal of entry into the glands.

1 Vitali C, Bombardieri S, Moutsopoulos HM, Balestrieri G, Bencivelli W, Bernstein RM, et al. Preliminary criteria for the classification of Sjogren's syndrome: results of a prospective concerted action supported by the European community. Arthritis Rheum 1993;36:340-7.

2 Fox RI, Robinson CA, Curd JG, Kozin F, Howell FV. Sjogren's syndrome: poposed criteria for classification. Arthritis Rheum 1986;29:577-85.

3 Skopouli FN, Fox PC, Galanopoulou V, Atkinson JC, Jaffe ES, Moutsopoulos HM. T cell subpopulations in the labial minor salivary gland histopathologic lesion of Sjogren's syndrome. J Rheumatol 1991;18:210-4.

4 Hedfors E, Lindahl G. Variation of MHC class I and II antigen expression in relation to lymphocytic infiltrates and gen expression in relation to lymphocytic infiltrates and
interferon gamma positive cells. J Rheumatol 1990; interferon gam $17: 743-50$.

5 Aziz KE, McCluskey PJ, Wakefield D. Expression of selectins (CD62 E,L,P) and cellular adhesion molecules in primary Sjogren's syndrome: questions to immunoregulation. Clin Immunol Immunopathol 1996;80:55-66.

6 Leroy JP, Pennec YL, Soulier C, Berthelot JM, Letoux G, Youinou P. Follow up study of labial salivary gland lesions in primary Sjogren's syndrome. Ann Rheum Dis 1992; 51:777-80.

7 Isenberg D. Sjogren's syndrome-a house of cards. Clin Rheumatol 1995;14(suppl 1):8-10.

8 Reeves WH. Immunopathogenesis of SLE and Sjogren's syndrome. Curr Opin Rheumatol 1990;2:717-23.

9 Aziz KE, Montanaro A, McCluskey PJ, Wakefield D. Sjogren's syndrome: review with recent insights into immunopathogenesis. Aust NZ J Med 1992;22:671-8.

10 Schriever F, Freedman AS, Freeman G, Messner E, Lee G, Daley J, et al. Isolated human follicular dendritic cells display a unique

11 Hart DNJ, McKenzie JL. Isolation and characterization of human tonsil dendritic cells. J Exp Med 1988;168:15769

12 Naiem M, Gerdes J, Abdulaziz Z, Stein H, Mason DY. Production of a monoclonal antibody reactive with human dendritic reticulum cells and its use in the immunohistological analysis of lymphoid tissue. J Clin Pathol 1983;36:167-75.

13 Carbone A, Poletti A, Manconi R, Gloghini A, Volpe R. Heterogeneous in situ immunophenotyping of follicular dendritic reticulum cells in malignant lymphomas of B-cell origin. Cancer 1987;60:2919-26

14 Heinen E, Bosseloir A. Follicular dendritic cells: whose children? Immunol Today 1994;15:201-4.

15 Thomas R, Lipsky PE. Human peripheral blood dendritic cell subsets. J Immunol 1994;153:4016-28.

16 Zutter MM, Santoro SA. Widespread histologic distribution of the $\alpha 2 \beta 1$ integrin cell surface collagen receptor. Am J Pathol 1990;137:113-20.

17 Chisholm DM, Mason DK. Labial salivary gland biopsy in Sjogren's syndrome. J Clin Pathol 1968;21:656-60.

18 Scheeren RA, Koopman G, Van der Baan S, Meijer CJLM, Pals S. Adhesion receptors involved in clustering of blood dendritic cells and $\mathrm{T}$ lymphocytes. Eur J Immunol $1991 ; 21: 1101-5$

19 Xu H, Friedrichs U, Gieseler RKH, Ruppert J, Ocklind G, Peters JH. Human blood dendritic cells exhibit a distinct T-cell stimulating mechanism and differentiation pattern. Scand J Immunol 1992;36:689-96. 